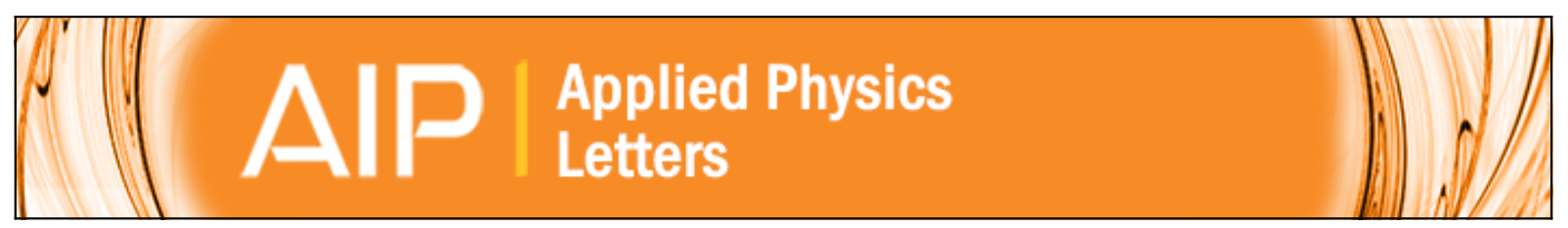

\title{
Extraordinary enhancement of Néel transition temperature in nanoparticles of multiferroic tetragonal compositions of (1-x)BiFeO3-xPbTiO3 solid solutions
}

Chandan Upadhyay, Pappu Kumar Harijan, Anatoliy Senyshyn, R. Ranganathan, and Dhananjai Pandey

Citation: Applied Physics Letters 106, 093103 (2015); doi: 10.1063/1.4913911

View online: http://dx.doi.org/10.1063/1.4913911

View Table of Contents: http://scitation.aip.org/content/aip/journal/apl/106/9?ver=pdfcov

Published by the AIP Publishing

\section{Articles you may be interested in}

Effect of Dy-substitution on the structural, vibrational, and multiferroic properties of BiFeO3 nanoparticles

J. Appl. Phys. 115, 214109 (2014); 10.1063/1.4881529

Structural and multiferroic properties of $\mathrm{Bi} 1-\mathrm{x} \ln x \mathrm{FeO} 3(0 \leq \mathrm{x} \leq 0.20)$ nanoparticles

J. Appl. Phys. 113, 044107 (2013); 10.1063/1.4788668

Effect of stress induced monoclinic to tetragonal phase transformation in the multiferroic (1-x)BiFeO3-xPbTiO3 system on the width of the morphotropic phase boundary and the tetragonality

J. Appl. Phys. 110, 084105 (2011); 10.1063/1.3647755

Simultaneous changes of nuclear and magnetic structures across the morphotropic phase boundary in $(1-\mathrm{x})$

$\mathrm{BiFeO} 3-\mathrm{xPbTiO} 3$

Appl. Phys. Lett. 97, 262506 (2010); 10.1063/1.3533665

Stability of the various crystallographic phases of the multiferroic $(1-\mathrm{x}) \mathrm{BiFeO} 3-\mathrm{xPbTiO} 3$ system as a function of composition and temperature

J. Appl. Phys. 107, 124112 (2010); 10.1063/1.3437396

\section{AIP $\mid$ APL Photonics}

APL Photonics is pleased to announce Benjamin Eggleton as its Editor-in-Chief

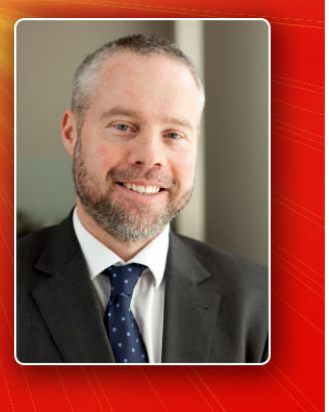




\title{
Extraordinary enhancement of Néel transition temperature in nanoparticles of multiferroic tetragonal compositions of $(1-\mathrm{x}) \mathrm{BiFeO}_{3}-\mathbf{x P b T i O}{ }_{3}$ solid solutions
}

\author{
Chandan Upadhyay, ${ }^{1}$ Pappu Kumar Harijan, ${ }^{1}$ Anatoliy Senyshyn, ${ }^{2}$ R. Ranganathan, ${ }^{3}$ \\ and Dhananjai Pandey ${ }^{1, a)}$ \\ ${ }^{1}$ School of Materials Science and Technology, Indian Institute of Technology (Banaras Hindu University), \\ Varanasi 221005, India \\ ${ }^{2}$ Forschungsneutronenquelle Heinze Maier-Leibnitz (FRM-II), Technische Universitat Munchen, \\ Liechtenbergestrass 1, D-85747 Garching b. Munchen, Germany \\ ${ }^{3}$ ECMP Division, Saha Institute of Nuclear Physics, I/AF Bidhannagar, Kolkata 700 064, India
}

(Received 7 January 2015; accepted 19 February 2015; published online 2 March 2015)

\begin{abstract}
The Néel temperature $\left(\mathrm{T}_{\mathrm{N}}\right)$ in conventional antiferromagnets (AFM) decreases with decreasing particle size. In contrast, we present here evidence for an exotic facet of multiferroicity, whereby one can raise the $T_{N}$ by more than $200 \mathrm{~K}$ by decreasing the particle size. We illustrate this by taking the example of a tetragonal composition with $\mathrm{x}=0.5$ in the solid solutions of $(1-\mathrm{x}) \mathrm{BiFeO}_{3}-\mathrm{xPbTiO}_{3}$ (BF-xPT). We attribute it to the increase in the strength of the superexchange interaction via a decrease in the ferroelectric distortion in nanocrystalline powder of BF-0.5PT. The BF-0.5PT nanoparticles also exhibit exchange bias effect due to AFM core-FM shell type magnetic nanostructure. (C) 2015 AIP Publishing LLC. [http://dx.doi.org/10.1063/1.4913911]
\end{abstract}

The properties of nanostructured magnetic materials differ significantly from their bulk counterparts and have been exploited for several technological applications. The nanostructured ferromagnetic (FM) and ferrimagnetic (FerM) materials are commonly used in permanent magnets, magnetic data storage, logic devices, ferrofluids, biomedical devices, etc. ${ }^{1-4}$ In recent years, nanostructured antiferromagnetic (AFM) materials have also received considerable attention for applications like spin valves, magnetic random access memory, and AFM and FM composite based hard magnets. ${ }^{5}$ Finite size effects in AFMs nanoparticles, nanorods, and thin films have been investigated extensively, ${ }^{6-10}$ and it has been shown that $\mathrm{T}_{\mathrm{N}}$ decreases with decreasing particle size or the thin film thickness as per the predictions of Néel. The decrease in $T_{N}$ is found to be consistent with Binder's scaling theory of critical phenomena in reduced dimension systems, which gives the following universal power law dependence of transition temperature: ${ }^{11}$

$$
\frac{T_{N}^{b}-T_{N}^{\text {grain }}}{T_{N}^{b}}=\left(\frac{d}{\xi_{0}}\right)^{-\left(\frac{1}{\nu}\right)}
$$

where $T_{N}^{b}$ and $T_{N}^{\text {grain }}$ are the bulk and finite size AFM transition temperatures, $d$ is the grain diameter, $\xi_{0}$ is the magnetic correlation length at $0 \mathrm{~K}$, and $\nu$ the critical exponent related to $\xi$.

In marked contrast to conventional AFMs materials, we show here that it is possible to raise the Néel temperature $\left(\mathrm{T}_{\mathrm{N}}\right.$ ) substantially (up to $\sim 200 \mathrm{~K}$ ) through particle size reduction in multiferroic AFM (MFAFM) materials. We also show that this extraordinary enhancement of $T_{N}$ in MFAFMs is linked with the tunablity of the strength of the

\footnotetext{
${ }^{\text {a) }}$ Author to whom correspondence should be addressed. Electronic mail: dp.mst1979@gmail.com. Telephone+91-9453048510
}

superexchange interaction through a decrease in the ferroelectric (FE) distortion in low dimensional systems due to finite size effects. We illustrate our finding using a tetragonal composition $\mathrm{x}=0.50$ of solid solutions of $\mathrm{BiFeO}_{3}$ with $\mathrm{PbTiO}_{3}$, i.e., $(1-\mathrm{x}) \mathrm{BiFeO}_{3}-\mathrm{xPbTiO}_{3}(\mathrm{BF}-0.5 \mathrm{PT})$, where $\mathrm{T}_{\mathrm{N}}$ is shown to increase from $\sim 120 \mathrm{~K}$ in bulk powders to $\sim 350 \mathrm{~K}$ in $18 \mathrm{~nm}$ size ferroelectric powders. While multiferroics are well known for magnetoelectric coupling involving controllability of electrical polarisation $(\mathrm{P})$ through magnetic field $(\mathrm{H})$ and magnetisation $(\mathrm{M})$ through electric field $(\mathrm{E})$ with potential for applications in new generation multifunctional sensor, actuator, and data storage devices, ${ }^{12-14}$ the present work brings out yet another exotic facet of multiferroicity, whereby one can raise $T_{N}$ systematically and design room temperature single phase multiferroics (which are extremely rare), useful for multifunctional devices operating at room temperature.

Amongst the magnetoelectric mutiferroic compounds, $\mathrm{BiFeO}_{3}(\mathrm{BF})$ is the only room temperature multiferroic with very high $\mathrm{FE}$ and $\mathrm{AFM}$ transition temperatures of $\mathrm{T}_{\mathrm{c}}=1103 \mathrm{~K}$ and $\mathrm{T}_{\mathrm{N}}=643 \mathrm{~K}$, respectively. ${ }^{12,15}$ This material therefore holds considerable promise for multifunctional devices operating at room temperature. ${ }^{12,13,15}$ Solid solutions of $\mathrm{BF}$ with other pervoskite oxides have received considerable attention from the point of view of release of latent magnetization from melting of the spin cycloid of BF. ${ }^{16} \mathrm{We}$ have considered the solid solution BF-XPT system for our study as it shows an interesting phase diagram having a critical point ${ }^{17}$ and a rich variety of structural and magnetic phase transitions like FE, antiferrodistortive (AFD), ${ }^{18}$ ferroelectric to ferroelectric isostructural, ${ }^{17}$ morphotropic, ${ }^{18,19}$ $\mathrm{AFM}^{20}{ }^{20}$ spin reorientation, ${ }^{21}$ and spin glass (SG) transitions. ${ }^{22} \mathrm{PbTiO}_{3}$ substitution modifies the rhombohedral R3c symmetry of $\mathrm{BF}$ to monoclinic Cc symmetry, ${ }^{18}$ similar to that in thin films of BF under epitaxial constraints. ${ }^{23,24}$ The 
monoclinic $\mathrm{Cc}$ phase is stable for $\mathrm{x} \leq 0.27$ and transforms to a tetragonal (space group $\mathrm{P} 4 \mathrm{~mm}$ ) phase for $\mathrm{x}=0.31$ via a thin phase coexistence region for $0.27<\mathrm{x}<0.31$ across a first order morphotropic phase boundary (MPB) ${ }^{18,19}$ The tetragonal phase of BF-xPT, stable for $0.31 \leq \mathrm{x} \leq 1.0$, has got several unusual characteristics such as (1) anomalously high tetragonality $(\eta=(\mathrm{c} / \mathrm{a}-1))$ that is more than three times that of the commercial MPB ceramics like PZT, ${ }^{19}$ (2) increasing tetragonality with increasing $\mathrm{BF}$ content on approaching the MPB and becoming as high as $\sim 18.73 \%$ for $\mathrm{x}=0.31^{18,19}$ ( $\eta$ decreases on approaching the MPB in PZT and other $\mathrm{PbTiO}_{3}$ based commercial MPB piezoceramics), (3) not resulting from the paraelectric (PE) cubic phase directly but through an isostructural phase transition from a low tetragonality $(\boldsymbol{\eta})$ phase, which gets formed first from the cubic paraelectric phase below the Curie temperature, ${ }^{17}$ and (4) possessing rather low $\mathrm{T}_{\mathrm{N}}(\sim 209 \mathrm{~K}$ for $\mathrm{x}=0.31)$ as compared to neighbouring monoclinic compositions with $\mathrm{T}_{\mathrm{N}} \sim 473 \mathrm{~K}$ for $\mathrm{x}=0.27 .{ }^{20} \mathrm{We}$ now proceed to show extraordinary enhancement of $T_{N}$ as a result of particle size reduction in tetragonal compositions of BF-xPT powders taking $\mathrm{x}=0.50$ (i.e., BF$0.5 \mathrm{PT}$ ) as a representative composition.

BF-0.50PT powders of approximate average sizes of $120 \mathrm{~nm}, 45 \mathrm{~nm}, 31 \mathrm{~nm}$, and $18 \mathrm{~nm}$ were synthesized by solgel route, the details of which are given in the supplementary material (Sec. A). ${ }^{25}$ Figure 1(a) shows the Synchrotron X-ray powder diffraction (SXRPD) profiles of the 100, 110, and 111 pseudocubic reflections of BF-0.5PT with average particle sizes of $\sim 18,31,45$, and $120 \mathrm{~nm}$. The SXRPD profiles of $120 \mathrm{~nm}$ size particles show that the 100 and 110 peaks are doublet, while 111 is a singlet as expected for the tetragonal phase with $\mathrm{P} 4 \mathrm{~mm}$ space group. With decreasing particle size, the splitting of 100 and 110 peaks decreases and the peak broadening increases. There is an asymmetric broadening of the 001 peak and other peaks with decreasing size. The $120 \mathrm{~nm}$ powder possesses a single tetragonal phase (space group $\mathrm{P} 4 \mathrm{~mm}$ ), whereas the smaller size powders show coexistence of two tetragonal phases, a dominant tetragonal phase with higher tetragonality, and a minority phase with relatively lower tetragonality, both in the $\mathrm{P} 4 \mathrm{~mm}$ space group, as confirmed by Rietveld refinement using SXRPD data. The details of Rietveld refinements are presented in the supplementary material (Sec. B). ${ }^{25}$ The $18 \mathrm{~nm}$ size particles do not show any obvious splitting of 200 and 220 and may appear to be cubic in the first appearance. However, Rietveld analysis of the SXRPD data (as explained in Sec. B of supplementary material) ${ }^{25}$ shows that the sample is still tetragonal with a tetragonality of $5.75 \%$ that is comparable to the tetragonality of $\mathrm{PbTiO}_{3}$. The variation of a and c parameters of BF-0.5PT, as obtained from Rietveld refinements, with particle size is shown in Fig. 1(b). The spontaneous polarisation of the dominant tetragonal phase of BF-0.5PT as calculated using Born effective charges of various cations and anions in $\mathrm{BF}^{26}$ and $\mathrm{PT}^{27}$ and the refined positional coordinates of BF-0.5PT are 32.1, 28.2, 24, and $23.1 \mu \mathrm{C} / \mathrm{cm}^{2}$ for $120,45,31$, and $18 \mathrm{~nm}$ size particles, respectively, confirming that significant ferroelectric polarization is present at room temperature even in the $18 \mathrm{~nm}$ particles. The reduction in tetragonality and spontaneous polarization with size is due to the well known "size effect" in ferroelectrics caused by
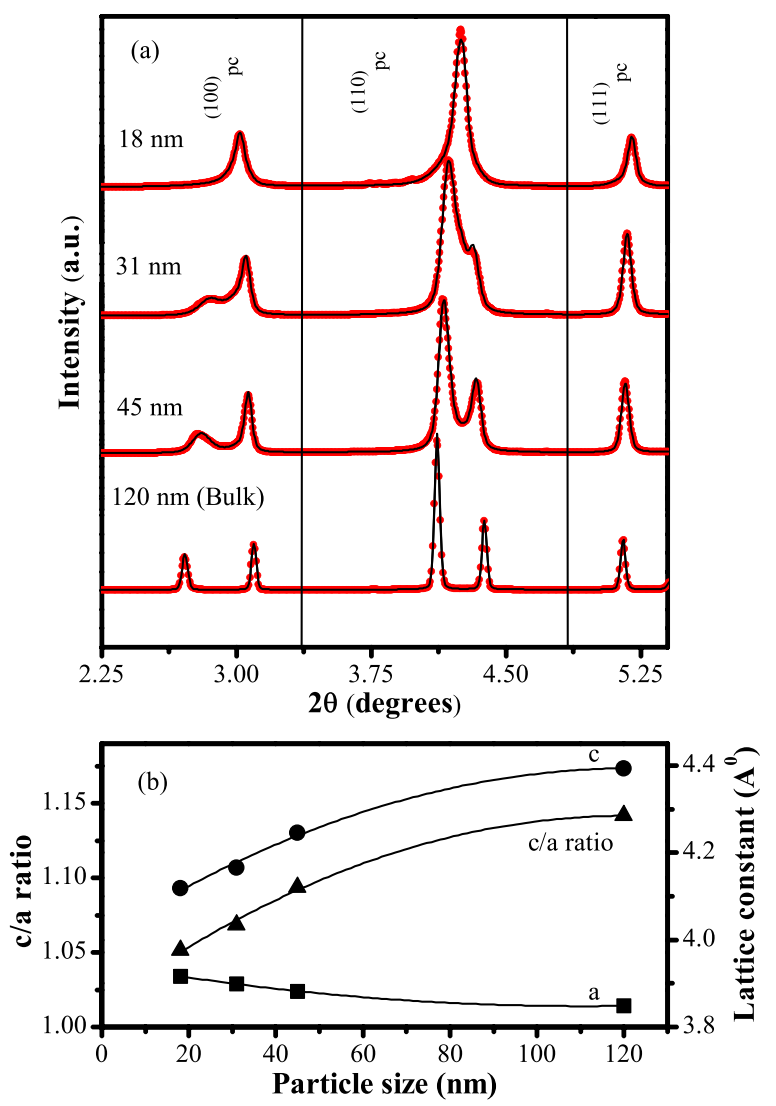

FIG. 1. (a) SXRPD profiles of 100, 110, and 111 pseudocubic reflections of BF-0.5PT samples with average particle sizes of $\sim 18,31,45$, and $120 \mathrm{~nm}$. (b) Variation of $\mathrm{c}$ and a lattice parameters and its ratio with particle size of BF-0.5PT.

the competition between depolarization and domain wall energies. $^{28}$

Figs. 2(a)-2(d) depict the results of magnetization (M) measurements as a function of temperature $(\mathrm{T})$ at an applied field of 5000 Oe for $120 \mathrm{~nm}, 45 \mathrm{~nm}, 31 \mathrm{~nm}$, and $18 \mathrm{~nm}$ size powders in zero-field cooled (ZFC) and field cooled (FC) modes. The ZFC M-T plot of the $120 \mathrm{~nm}$ size particles shows a Néel transition temperature $T_{N} \sim 120 \mathrm{~K}$ (see the inset also) similar to that reported in bulk powders of BF-0.5PT. ${ }^{11}$ Intriguingly, on reducing the particle size to 45,31 , and $18 \mathrm{~nm}$, the Néel temperature increases drastically to 230 , 250 , and $350 \mathrm{~K}$, respectively, as can be seen from various insets to Fig. 2. The anomaly at $\mathrm{T}_{\mathrm{f}} \sim 17 \mathrm{~K}$ in the ZFC M-T plot of the bulk is known to be due to spin glass freezing. ${ }^{22}$

The enhancement of $T_{N}$ revealed by $M(T)$ measurements was confirmed by neutron powder diffraction studies as well. The temperature evolution of the magnetic peak corresponding to propagation vector $\mathbf{k}=1 / 21 / 21 / 2$ in the neutron powder diffraction patterns is shown in Figs. 3(a) and 3(b) for bulk and $18 \mathrm{~nm}$ size powders, respectively. In the bulk samples, the AFM peak (marked with asterisk in the figure) appears below $\mathrm{T}_{\mathrm{N}} \sim 120 \mathrm{~K}$, whereas it is present even at room temperature in $18 \mathrm{~nm}$ size powder. This clearly shows a drastic enhancement of $T_{N}$ in $18 \mathrm{~nm}$ size powder. The variation of the integrated intensity of the $1 / 21 / 21 / 2$ magnetic peak with temperature shown in Fig. 3(c) reveals $\mathrm{T}_{\mathrm{N}} \sim 120 \mathrm{~K}$ in agreement with the $\mathrm{T}_{\mathrm{N}}$ determined from ZFC M-T measurements (Fig. 2(a)). The magnetic neutron peaks of $18 \mathrm{~nm}$ particles 


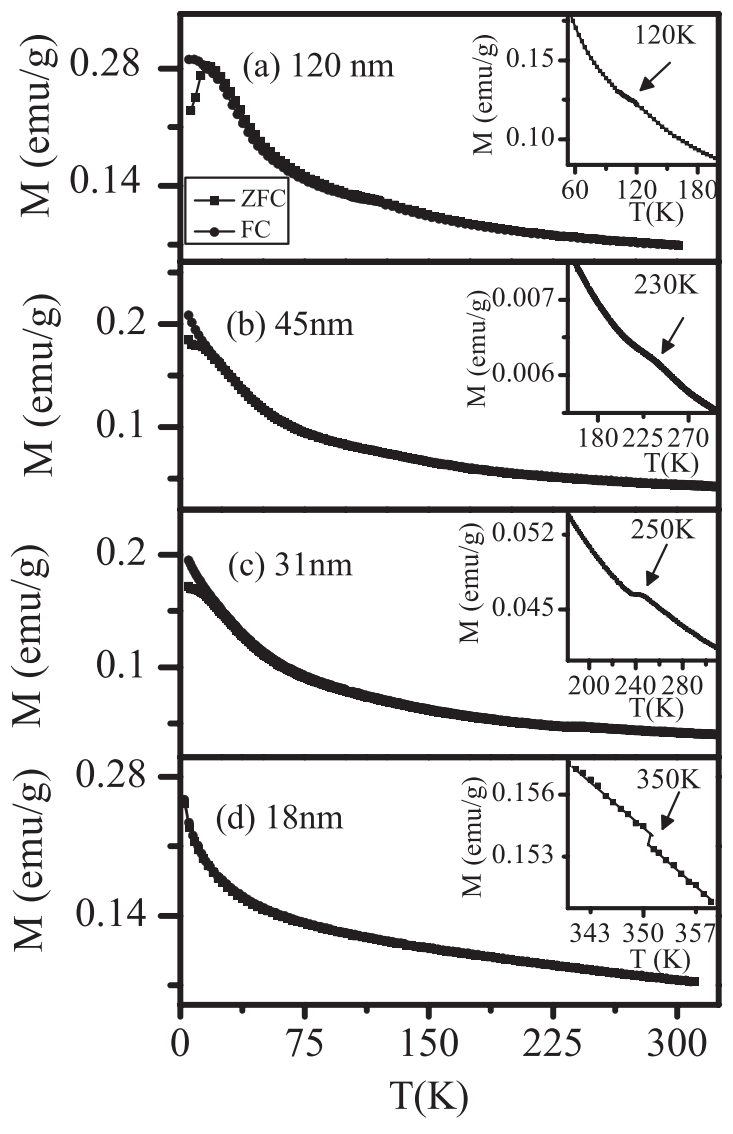

FIG. 2. The temperature dependence of the FC and ZFC magnetizations of BF-0.5PT: (a-d) 120, 45, 31 and $18 \mathrm{~nm}$ size powders under magnetic field of 5000 Oe, The insets of (a to d) depict magnified plot near $T_{N}$.

have sharp and diffuse (broad) components (see Fig. 3(d) which depicts two deconvoluted Lorentzians) linked with the coexisting high and low tetragonality phases, respectively, as confirmed by Rietveld refinements using SXRPD data (see Sec. B of supplementary material). ${ }^{25}$ The diffuse component of the magnetic scattering is dominant above $250 \mathrm{~K}$. The variation of the integrated intensity of the sharp component with temperature for $18 \mathrm{~nm}$ size powder shows a linear behaviour up to $\sim 250 \mathrm{~K}$ and its extrapolation to zero intensity gives a $\mathrm{T}_{\mathrm{N}}$ of $\sim 350 \mathrm{~K}$ in agreement with the $\mathrm{M}(\mathrm{T})$ results. Thus, there is a drastic enhancement of $T_{N}$ with respect to $T_{N}=120 \mathrm{~K}$ of the bulk powder. The diffuse component shows a temperature dependence different from that of the sharp component and the corresponding $T_{N}$ is still higher, as small residual intensity can be seen at the AFM peak position even in 360 and $400 \mathrm{~K}$ profile in Fig. 3(b).

We propose that the diffuse component of the magnetic peak with shorter correlation length is due to spin disorder in a thin shell region of the surface layers of the nanoparticles. Both the shell and the core contain the BF-0.50PT solid solution only. In this scenario, one expects weak ferromagnetism due to spin canting in the shell region of the $18 \mathrm{~nm}$ size nanoparticles of BF-0.5PT as observed in similar core-shell type systems in non multiferroic AFM oxides. ${ }^{29}$ This weak ferromagnetism should be present even at room temperature as the corresponding $\mathrm{T}_{\mathrm{N}}$ is well above the room temperature for $18 \mathrm{~nm}$ size particles. This was confirmed by the M-H measurements, the results of which are shown in
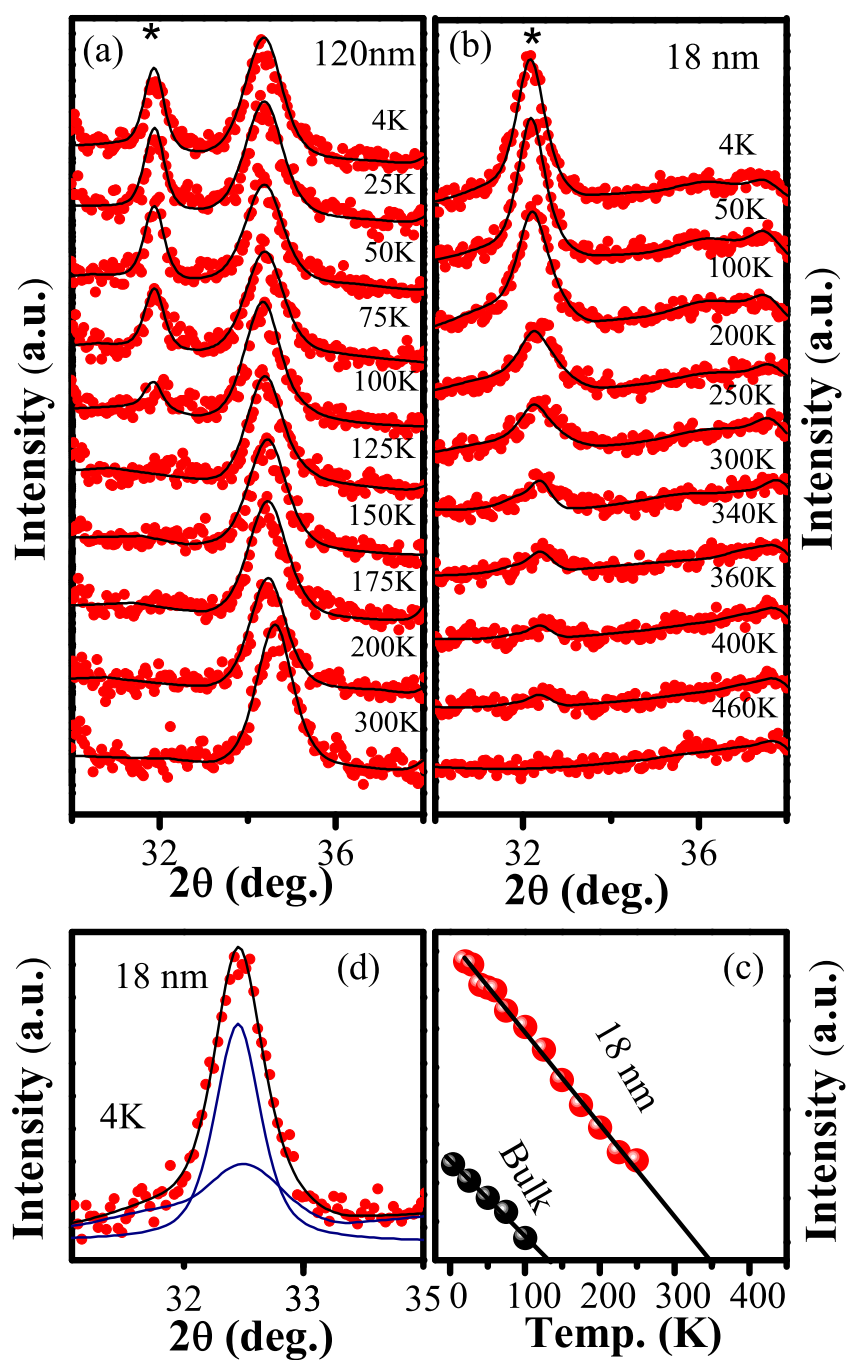

FIG. 3. The evolution of the magnetic peak corresponding to pseudocubic $\mathrm{k}=1 / 21 / 21 / 2$ with temperature for (a) 120 and (b) $18 \mathrm{~nm}$ size powders of BF0.5PT. (c) Variation in the integrated intensity of the magnetic peak with temperature for 120 and $18 \mathrm{~nm}$ size powders. Dots represent measured data points, while the continuous line corresponds to the least-squares fitted Lorentzian(s). (d) Deconvolution of the magnetic peak of $18 \mathrm{~nm}$ size powder showing two different magnetic correlation lengths $(\xi)$.

Fig. 4. It is evident from Fig. 4(a) that the M-H plots for $120 \mathrm{~nm}, 45 \mathrm{~nm}$, and $31 \mathrm{~nm}$ size powders are straight lines confirming their paramagnetic nature at room temperature, whereas the $18 \mathrm{~nm}$ size particles exhibit nonlinear M-H curve, with a little loop opening. With decreasing temperature, the M-H loop of $18 \mathrm{~nm}$ size particles opens up further as can be seen from the two insets of Fig. 4(b), which compare the M-H plots for $18 \mathrm{~nm}$ size particles at $300 \mathrm{~K}$ and $5 \mathrm{~K}$. Further, the M-H loop in inset of Fig. 4(b) reveals an exchange bias $\left(\mathrm{E}_{\mathrm{B}}\right)$ of $\sim 337 \mathrm{Oe}$ at $5 \mathrm{~K}$, which decreases with increasing temperature for the same particle size. The $\mathrm{E}_{\mathrm{B}}$ decreases with increasing particle size also for the same temperature. For example, the $\mathrm{E}_{\mathrm{B}}$ at $5 \mathrm{~K}$ is $\sim 17 \mathrm{Oe}$ and $\sim 8$ Oe for $31 \mathrm{~nm}$ and $45 \mathrm{~nm}$ size powders, respectively, while it is absent for the bulk powder (see inset of Fig. $4(\mathrm{c}))$. The more pronounced $\mathrm{E}_{\mathrm{B}}$, as shown in the inset of Fig. 4(b), in the smallest size particles $(\sim 18 \mathrm{~nm})$, is due to higher surface to volume ratio of $18 \mathrm{~nm}$ particles leading to more number of spins in the surface layers constituting the shell region. The interface between the FM component of 


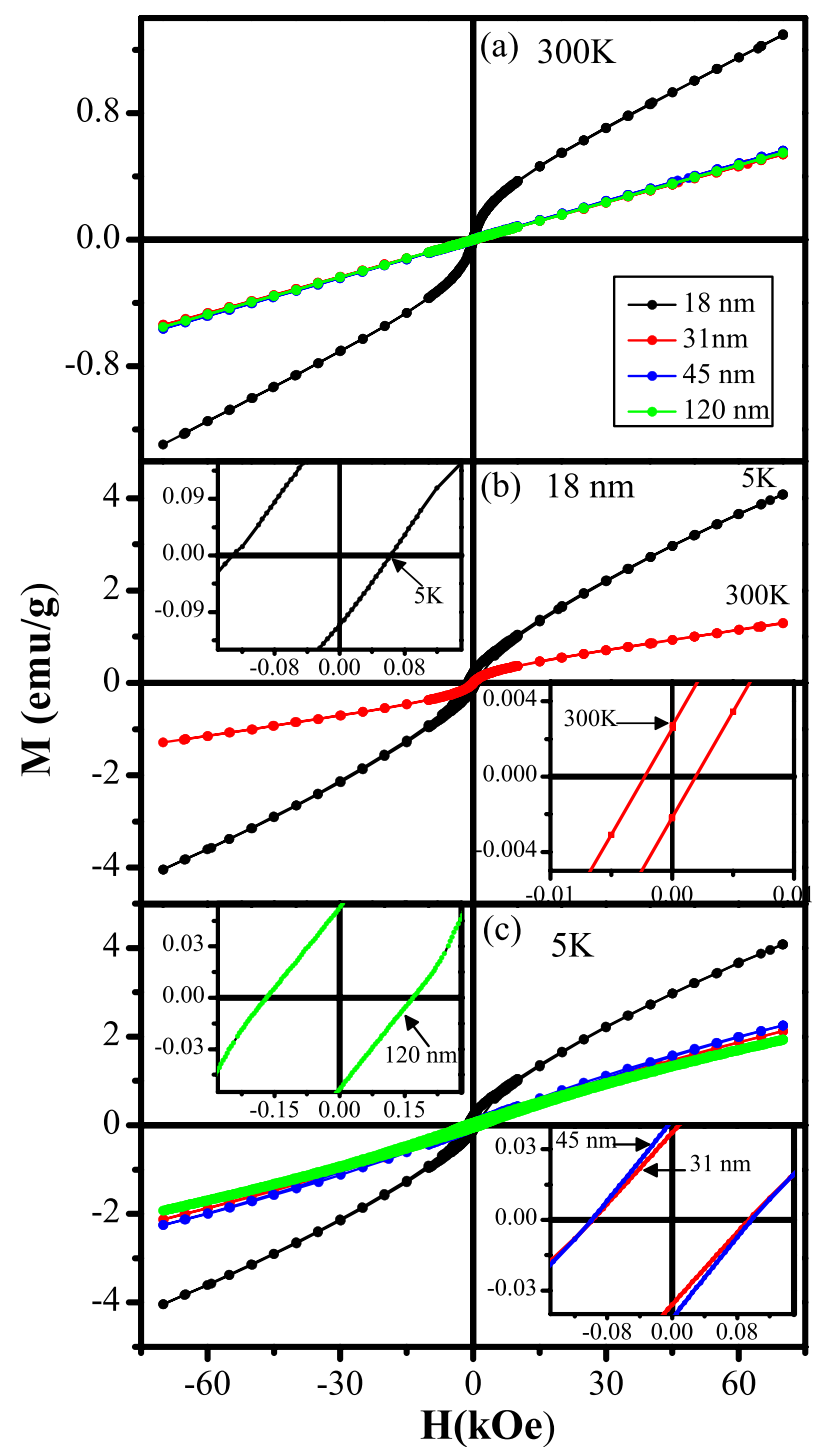

FIG. 4. M-H plots for (a) 18, 31, 45, and $120 \mathrm{~nm}$ size powders at $300 \mathrm{~K}$, (b) $18 \mathrm{~nm}$ particles at 300 and $5 \mathrm{~K}$ and (c) $18,31,45$, and $120 \mathrm{~nm}$ size powders at $5 \mathrm{~K}$. Insets of (b) and (c) compare exchange bias at $5 \mathrm{~K}$ and $300 \mathrm{~K}$ for $18 \mathrm{~nm}$ and at $5 \mathrm{~K}$ for 120 and $31 \mathrm{~nm}$ powders.

the canted spins in the shell region and the collinear AFM spins in the core (of BF-0.50PT) is responsible for the exchange bias effect as observed in other systems also. ${ }^{29-31}$ Thus, our $\mathrm{M}-\mathrm{H}$ plot showing $\mathrm{E}_{\mathrm{B}}$ effect not only confirms the coexistence of AFM and weak FM ordering but also reveals that they arise from a core-shell type of nanostructure of individual nanoparticles.

The enhancement of $T_{N}$ with size reduction is unique to MFAFMs with large uniaxial ferroelectric distortion like tetragonal BF-0.5PT. It is a consequence of the subtle interplay between the ferroelectric distortion and the strength of the superexchange interaction in the multiferroic BF-0.5PT. Table I lists the lengths of $\mathrm{Fe}^{3+}-\mathrm{O}^{2-}$ bond lengths in the [001] direction for the bulk and the $18 \mathrm{~nm}$ size powder, as calculated using the positional coordinates obtained by Rietveld analysis of SXRPD patterns. The longer $\mathrm{Fe}^{3+}-\mathrm{O}^{2-}$ bond length (labelled as $\mathrm{Fe}-\mathrm{O}_{1 \mathrm{~b}}$ in Table I) in the bulk powder is too big $(2.571 \AA)$ as compared to the sum of the ionic radii of $\mathrm{Fe}^{3+}$ and $\mathrm{O}^{2-}(\sim 2.045 \AA)$ to have any orbital overlap required for superexchange interactions in [001] direction in
TABLE I. Fe-O bond lengths $(\AA)$ and tetragonality of the dominant phase constituting the core of BF-0.5PT samples as a function of particle size.

\begin{tabular}{lcccc}
\hline \hline & & \multicolumn{3}{c}{ Bond length $(\AA)$} \\
\cline { 3 - 5 } Size $(\mathrm{nm})$ & Tetragonality $(\eta) \%$ & $\mathrm{Fe}-\mathrm{O}_{1 \mathrm{a}}$ & $\mathrm{Fe}-\mathrm{O}_{1 \mathrm{~b}}$ & $\mathrm{Fe}-\mathrm{O}_{2}$ \\
\hline 18 & 5.75 & 1.787 & 2.348 & 2.013 \\
31 & 6.64 & 1.790 & 2.369 & 1.970 \\
45 & 9.60 & 1.792 & 2.460 & 1.973 \\
120 & 14.16 & 1.822 & 2.571 & 1.968 \\
\hline \hline
\end{tabular}

the bulk powders. As a result, the AFM superexchange interaction pathways are confined to [100] and [010] directions only. As the longer $\mathrm{Fe}^{3+}-\mathrm{O}^{2-}$ bond in the [001] direction decreases systematically with particle size reduction (see Table I for bond length of the $18 \mathrm{~nm}$ size powder), there is a gradual enhancement of superexchange interaction in the [001] direction also. This leads to a crossover from essentially $2 \mathrm{~d}$ AFM interactions in the bulk powders to $3 \mathrm{~d}$ AFM interactions in nanoparticles of BF-0.50PT and it is this crossover that is responsible for the drastic rise in the $T_{N}$ as a result of size reduction. It is interesting to note that such an enhancement of $T_{N}$ with decreasing particle size does not happen in $\mathrm{BiFeO}_{3}$ where $\mathrm{T}_{\mathrm{N}}$ instead decreases with particle size as per the predictions of the scaling theories. ${ }^{32}$ This is because there is no crossover from $2 \mathrm{~d}$ AFM to $3 \mathrm{~d}$ AFM interactions in $\mathrm{BiFeO}_{3}$ due to particle size reduction, as $\mathrm{BF}$ shows 3d AFM interactions in the bulk itself. $\mathrm{Ti}^{4+}$ substitution decreases the $T_{N}$ as result of dilution of the magnetic sublattice of BF monotonically up to $\mathrm{x} \sim 0.27$ but drops abruptly in the tetragonal phase region of BF-xPT for compositions with $\mathrm{x} \geq 0.31$ because of the crossover from $\sim 3 \mathrm{~d}$ AFM interactions for $\mathrm{x} \leq 0.27$ to $\sim 2 \mathrm{~d}$ interactions for $\mathrm{x} \geq 0.31$ as a result of large anisotropic ferroelectric distortion in the tetragonal structure. ${ }^{20}$ For example, the experimentally observed $\mathrm{T}_{\mathrm{N}}$ $\approx 120 \mathrm{~K}$ for bulk BF-0.5PT powders is far less than the expected value of $\mathrm{T}_{\mathrm{N}} \approx 365 \mathrm{~K}$ for $3 \mathrm{~d} A F M$ interactions obtained by extrapolation of $\mathrm{T}_{\mathrm{N}}$ versus $\mathrm{x}$ plot for BF-xPT with $\mathrm{x} \leq 0.27$ with monoclinic structure to $\mathrm{x}=0.50$ in the tetragonal phase region. On the other hand, $\mathrm{T}_{\mathrm{N}} \sim 350 \mathrm{~K}$ of $\sim 18 \mathrm{~nm}$ size BF-0.50PT particles is comparable to the estimated value of $\mathrm{T}_{\mathrm{N}} \sim 365 \mathrm{~K}$ for $3 \mathrm{~d}$ AFM interactions in BF$0.5 P T$. For $\sim 18 \mathrm{~nm}$ size particles, $\mathrm{T}_{\mathrm{N}}$ of $\mathrm{BF}$ decreases substantially due to purely size effect, ${ }^{33}$ whereas that of BF$0.5 \mathrm{PT}$ increases substantially due to crystal distortion effect, but interestingly both become comparable in spite of magnetic lattice being diluted by $50 \%$ with $\mathrm{Ti}^{4+}$ in the latter. In this respect, BF-0.5PT nanoparticles are superior to BF nanoparticles but the ferroelectric polarization and magnetization appear to be larger for BF nanoparticles.

To summarize, our results reveal that the effect of decrease in AFM correlation length with particle size leading to reduction in $T_{N}$ as per scaling theories is overwhelmed by the enhancement in the superexchange interactions due to crystal distortion effects leading to reduced bond length in nanoparticles of BF-0.50PT in the [001] direction. Thus, the physics of size effect in multiferroic AFM systems with large uniaxial ferroelectric distortion is entirely different from that in conventional AFM systems with 3d AFM interactions. We believe that our results will stimulate more work as it has 
tremendous potential for designing room temperature multiferroics useful for multifunctional devices by tuning the $T_{N}$ to room temperature through a manipulation of the ferroelectric distortion via size reduction.

D. Pandey acknowledges financial support from Science and Engineering Research Board (SERB) of India through J. C. Bose Fellowship grant. P. K. Harijan acknowledges financial support under Rajiv Gandhi National Fellowship (RGNF) programme of India. We acknowledge the assistance of Dr. M. Hinterstein from PETRA III, DESY, Germany, in collection of SXRPD data. We also acknowledge financial support from Saha Institute of Nuclear Physics (SINP) under the "India-DESY Germany" project to carry out SXRPD experiments at DESY, Germany.

${ }^{1}$ J. L. Dormann, D. Fiorani, and E. Tronc, Adv. Chem. Phys. 98, 283 (1997).

${ }^{2}$ Magnetic Properties of Fine Particles, edited by J. L. Dormann and D. Fiorani (North-Holland, Amsterdam, 1992).

${ }^{3}$ Surface Effects in Magnetic Nanoparticles, edited by D. Fiorani (Springer, New York, 2005).

${ }^{4}$ Q. A. Pankhurst, J. Conolly, S. K. Jones, and J. Dobson, J. Phys. D: Appl. Phys. 36, R167 (2003).

${ }^{5}$ S. Mørup, D. E. Madsen, C. Frandsen, C. R. H. Bahl, and M. F. Hansen, J. Phys.: Condens. Matter 19, 213202 (2007).

${ }^{6}$ M. Molina-Ruiz, A. F. Lopeandía, F. Pi, D. Givord, O. Bourgeois, and J. Rodríguez-Viejo, Phys. Rev. B 83, 140407(R) (2011).

${ }^{7}$ R. H. Kodma, A. Makhlouf Salah, and A. E. Berkowitz, Phys. Rev. Lett. 79, 1393 (1997).

${ }^{8}$ T. Ambrose and C. L. Chien, Phys. Rev. Lett. 76, 1743 (1996).

${ }^{9}$ Y. J. Tang, D. J. Smith, B. L. Zink, F. Hellman, and A. E. Berkowitz, Phys. Rev. B 67, 054408 (2003).

${ }^{10}$ E. Weschke, H. Ott, E. Schierle, C. Schüßler-Langeheine, D. V. Vyalikh, G. Kaindl, V. Leiner, M. Ay, T. Schmitte, H. Zabel et al., Phys. Rev. Lett. 93, 157204 (2004).
${ }^{11}$ K. Binder, Physica 62, 508 (1972).

${ }^{12}$ W. Eerenstein, N. D. Mathur, and J. F. Scott, Nature (London) 442, 759 (2006).

${ }^{13}$ S.-W. Cheong and M. Mostovoy, Nat. Mater. 6, 13 (2007).

${ }^{14}$ R. Ramesh and N. A. Spaldin, Nat. Mater. 6, 21 (2007).

${ }^{15}$ M. Fiebig, J. Phys. D 38, R123 (2005).

${ }^{16}$ A. Singh, V. Pandey, R. K. Kotnala, and D. Pandey, Phys. Rev. Lett. 101, 247602 (2008).

${ }^{17}$ S. Bhattacharjee, K. Taji, C. Moriyoshi, Y. Kuroiwa, and D. Pandey, Phys. Rev. B 84, 104116 (2011).

${ }^{18}$ S. Bhattacharjee and D. Pandey, J. Appl. Phys. 107, 124112 (2010).

${ }^{19}$ S. Bhattacharjee, S. Tripathi, and D. Pandey, Appl. Phys. Lett. 91, 042903 (2007).

${ }^{20}$ S. Bhattacharjee, A. Senyshyn, P. S. R. Krishna, H. Fuess, and D. Pandey, Appl. Phys. Lett. 97, 262506 (2010).

${ }^{21}$ S. Bhattacharjee, A. Senyshyn, H. Fuess, and D. Pandey, Phys. Rev. B 87, 054417 (2013).

${ }^{22}$ W.-M. Zhu, H.-Y. Guo, and Z.-G. Ye, Phys. Rev. B 78, 014401 (2008).

${ }^{23}$ R. J. Zeches, M. D. Rossell, J. X. Zhang, A. J. Hatt, Q. He, C.-H. Yang, A. Kumar, C. H. Wang, A. Melville, C. Adamo et al., Science 326, 977 (2009).

${ }^{24}$ S. Bhattacharjee and D. Pandey, J. Appl. Phys. 110, 084105 (2011).

${ }^{25}$ See supplementary material at http://dx.doi.org/10.1063/1.4913911 for details of sample synthesis and for structure refinement using synchrotron $\mathrm{X}$-ray powder diffraction data.

${ }^{26}$ P. Ravindran, R. Vidya, A. Kjekshus, and H. Fjellvåg, Phys. Rev. B 74, 224412 (2006).

${ }^{27}$ G. S. Szabó, R. E. Cohen, and H. Krakauer, Phys. Rev. Lett. 80, 4321 (1998).

${ }^{28}$ C. H. Ahn, K. M. Rabe, and J. M. Triscone, Science 303, 488 (2004).

${ }^{29}$ M. J. Benitez, O. Petracic, E. L. Salabas, F. Radu, H. Tüysüz, F. Schüth, and H. Zabel, Phys. Rev. Lett. 101, 097206 (2008).

${ }^{30}$ S. M. Yusuf, P. K. Manna, M. M. Shirolkar, S. K. Kulkarni, R. Tewari, and G. K. Dey, J. Appl. Phys. 113, 173906 (2013).

${ }^{31}$ P. K. Manna and S. M. Yusuf, Phys. Rep. 535, 61 (2014).

${ }^{32}$ S. M. Selbach, T. Tybell, M.-A. Einarsrud, and T. Grande, Chem. Mater. 19, 6478-6484 (2007).

${ }^{33}$ S. Goswami, D. Bhattacharya, and P. Choudhary, J. Appl. Phys. 109, 07D737 (2011). 